\title{
Insectos asociados a cultivos de pitaya amarilla (Selenicereus megalanthus) en Inzá, Cauca, Colombia
}

\author{
Insects associated to yellow pitaya crops (Selenicereus megalanthus) on \\ Inzá, Cauca, Colombia \\ MARÍA DEL MAR GONZÁLEZ-TRUJILLO ${ }^{1}$; ANDRÉS RICARDO PERAZA ARIAS²; \\ HELENA L. BROCHERO ${ }^{3}$
}

\begin{abstract}
${ }^{1}$ Ingeniera Agrónoma, Facultad de Ciencias Agrarias, Universidad Nacional de Colombia, sede Bogotá, Bogotá, Colombia, mmgonzalezt@unal.edu.co, https:// orcid.org/0000-0003-4299-2653. ${ }^{2}$ M. Sc. Ciencias Agrarias, Entomología, Universidad Nacional de Colombia, sede Bogotá, Bogotá, Colombia, arperazaa@ unal.edu.co, https://orcid.org/0000-0003-3421-5694. ${ }^{3}$ Docente, Facultad de Ciencias Agrarias, Universidad Nacional de Colombia, sede Bogotá, Bogotá, Colombia, embrochero@unal.edu.co, https://orcid.org/0000-0002-0325-5661.
\end{abstract}

Autor de correspondencia: María del Mar González-Trujillo. Ingeniera Agrónoma, Facultad de Ciencias Agrarias, Universidad Nacional de Colombia, sede Bogotá, Bogotá, Colombia,mmgonzalezt@unal.edu.co,https:// orcid.org/0000-0003-4299-2653

Citación sugerida / Suggested citation: GONZÁLEZ-TRUJILLO, M. D. M.; PERAZA ARIAS, A. R.; BROCHERO, H. L. 2019. Insectos asociados a cultivos de pitaya amarilla (Selenicereus megalanthus) en Inzá, Cauca, Colombia. Revista Colombiana de Entomología 45 (2): e7961. https://doi.org/10.25100/socolen. v45i2.7961

Recibido: 26-mar-2018

Aceptado: 18-mar-2019

Publicado: 17-ene-2020

Revista Colombiana de Entomología ISSN (Impreso): 0120-0488

ISSN (En línea): 2665-4385

$\mathrm{http}: / /$ revistacolombianaentomologia.univalle.edu.co/

Open access

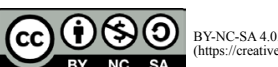

Publicadores / Publishers:

Sociedad Colombiana de Entomología SOCOLEN (Bogotá, D. C., Colombia) http://www.socolen.org.co Universidad del Valle (Cali, Colombia) http://www.univalle.edu.co/

(C) 2019 Sociedad Colombiana de Entomología - SOCOLEN y Universidad del Valle - Univalle
Resumen: Como alternativa al descenso en la rentabilidad del cultivo de café, principal fuente económica en el municipio de Inzá (Cauca, Colombia), se establecieron cultivos de pitaya amarilla sin conocer las limitantes fitosanitarias para este cultivo en esta zona. Este trabajo presenta la entomofauna asociada a cultivos de pitaya amarilla en tres predios productivos de Inzá, contrastantes por sus características agronómicas y de paisaje. Se realizó muestreo directo de insectos sobre cada estructura de la planta, muestreo con pases dobles de red entomológica a $50 \mathrm{~cm}$ de distancia de la planta y se consideraron factores abióticos e índices de diversidad ecológica. Con muestreo directo se reconocieron 58 familias, prevaleciendo Formicidae $(\mathrm{n}=$ $22)$, Coreidae $(n=11)$ y Lonchaeidae $(n=7)$. Con red entomológica se recolectaron cinco órdenes y 56 familias de insectos. Las plantas con mayor densidad de cladodios favorecieron a las familias Lonchaeidae $(\tau=1,0 ; \mathrm{P}=0,00)$, Formicidae $(\tau=1,00 ; \mathrm{P}=0,007)$ y Cicadellidae $(\tau=1,00 ; \mathrm{P}=0,00)$. La precipitación $(\tau=1,00 ; \mathrm{P}=0,00)$ y la temperatura $(\tau=1,00 ; \mathrm{P}=$ $0,00)$ se correlacionaron positivamente beneficiando a Lonchaeidae. No se encontraron daños asociados a Dasiops spp. (Diptera). No se confirmó la presencia de D. saltans porque todos los especímenes correspondieron a machos. Los índices de diversidad de Margalef $\left(\mathrm{D}_{\mathrm{M}}\right)=$ 7,42; Shannon-Wiener $\left(\mathrm{H}^{\prime}\right)=3,217$ (Alfa) y Whittaker $(\beta \mathrm{w})=1,0$; y Routledge $(\beta \mathrm{r})=0,25$ (Beta) representados principalmente por gran variedad de familias de insectos benéficos. Debe establecerse un sistema de vigilancia entomológica regular y acompañamiento técnico a los productores con miras al sostenimiento económico a largo plazo para este cultivo en el municipio.

Palabras clave: Dasiops, ecología, relaciones planta-insecto, Colombia, región Neotropical.

Abstract: In the municipality of Inzá (Cauca, Colombia) the profitability of the main economic resource, coffee crops, decreased. As an alternative, yellow pitaya crops were established, but knowledge of the possible phytosanitary limitations for this crop in this region was absent. This study presents the entomofauna associated with yellow pitaya crops in three plantations of Inzá that contrast in agronomic and landscape features. Direct sampling of the insects found on each structure of the plant and sampling was done using entomological nets from directly under the plants to $50 \mathrm{~cm}$ away. Abiotic factors and indices of ecological diversity were considered. With direct sampling, 58 families were recognized; of these, Formicidae $(n=22)$, Coreidae $(n=11)$ and Lonchaeidae $(n=7)$ were most prevalent. Five orders and 56 families of insects were collected using entomological nets. The plants with the highest density of cladodes favored the Lonchaeidae $(\tau=1.0 ; \mathrm{P}=0.00)$, Formicidae $(\tau=1.00 ; \mathrm{P}=$ $0.007)$ and Cicadellidae $(\tau=1.00 ; P=0.00)$ families. Insects from the Lonchaeidae family benefitted from the positive correlation between rainfall $(\tau=1.00 ; \mathrm{P}=0.00)$ and temperature $(\tau=1.00 ; \mathrm{P}=0.00)$. Damage associated with Dasiops spp. (Diptera) was not found. The presence of D. saltans was not confirmed because all the specimens collected were males. The Margalef $(\mathrm{DMg})=7.42$; Shannon-Wiener $\left(\mathrm{H}^{\circ}\right)=3.217$ (Alpha) and Whittaker $(\beta \mathrm{w})=$ 1.0 ; and Routledge $(\beta r)=0.25$ (Beta) diversity indices represented a large variety of beneficial insect families. A system of regular entomological surveillance and technical support should be established for producers with aim of providing long-term economic viability for this crop in the municipality.

Keywords: Dasiops, ecology, plant-insect relationships, Colombia, Neotropical region.

\section{Introducción}

Las pitayas o pitahayas forman parte del orden Cactales, familia Cactaceae, subfamilia Cereoideae, tribu Hylocereae, subtribu Hylocereinae, con 
representación de dos géneros de importancia agrícola: Hylocereus (Berger) Br y R., que corresponde a frutos de epidermis roja y Selenicereus (Berger) Br. y R., con frutos tetraploides de epidermis amarilla (Nobel 2002; Rodríguez 2002) y con por lo menos, 20 especies reconocidas (Barthlott y Hunt 1993; Tel-Zur et al. 2004). S. megalanthus Bauer (K Schumann ex Vaupel) Moran 1953, comúnmente conocida como pitaya amarilla, tiene su origen en Colombia, Perú, Bolivia, Ecuador y Venezuela; corresponde a plantas perennes, terrestres trepadoras y epifitas, que requieren de soporte para su producción comercial. Su propagación comercial es vegetativa a partir de tallos o esquejes, la producción de frutos inicia entre uno a dos años después del establecimiento y puede tener una vida productiva mayor a 10 años (Rodríguez 2002).

Colombia es el principal productor y exportador de pitaya amarilla a nivel mundial, con áreas sembradas en Boyacá, Valle del Cauca, Bolívar, Caldas, Cundinamarca, Huila, Quindío, Risaralda, Santander y Tolima (Betancourt et al. 2010). Las zonas más adecuadas para el cultivo se encuentran entre 1.400 y $1.700 \mathrm{msnm}$, con temperaturas entre 14 a $26^{\circ} \mathrm{C}$, suelos con $\mathrm{pH}$ entre 5,5 y 6,5 y precipitaciones de 1.300 a 2.200 $\mathrm{mm}$ anuales (Rebolledo et al. 2003). Aunque el municipio de Inzá, Cauca se considera zona ecológica marginal para este cultivo, se establecieron de novo cultivos de pitaya amarilla a partir de 2015, como alternativa de diversificación y como una respuesta a la reducción de la rentabilidad del cultivo de café, tradicionalmente sustento económico. A pesar de los altos costos para el establecimiento de este cultivo, el proceso se llevó a cabo sin asistencia técnica o estudios que permitieran estimar las limitantes para su producción y comercialización. El objetivo de este estudio fue caracterizar la entomofauna asociada a cultivos de pitaya amarilla de tres predios productivos, con características de la composición del paisaje agrícola contrastantes, del municipio de Inzá, Cauca, Colombia.

\section{Materiales y métodos}

Sitio de estudio. El municipio de Inzá, Cauca, se encuentra ubicado a los $02^{\circ} 33^{\prime} 24^{\prime \prime} \mathrm{N}$ y $76^{\circ} 04^{\prime} 00^{\prime \prime O}$; con cabecera municipal ubicada a una altitud de $1.800 \mathrm{msnm}$, temperatura promedio anual de $19{ }^{\circ} \mathrm{C}$ y precipitación media anual de $1.526 \mathrm{~mm}$ (Gobernación del Cauca 2013). El estudio se llevó a cabo en tres predios productivos para pitaya amarilla localizados en distintas zonas altitudinales y con diferentes arreglos agronómicos de producción. 1) Predio Piedra Mesa $\left(02^{\circ} 30^{\prime} 10,63^{\prime} \mathrm{N}, 076^{\circ} 02^{\prime} 58,22^{\prime}\right.$ 'O) de la vereda Turminá, a una altitud de $1.804 \mathrm{msnm}$, con el cultivo pitaya amarilla en pendiente de $33 \%$ y tutorado vivo de árboles maderables como flor amarillo (Handroanthus chrysanthus (Jacq.) S, O. Grose, 1797) combinados con alambre y cultivos transitorios como maíz (Zea mays L., 1753). En la periferia, el cultivo está rodeado de árboles de mandarina (Citrus reticulata Blanco, 1837) y aguacate (Persea americana Mill., 1768). 2) Predio Buena Vista $\left(02^{\circ} 31^{\prime} 33,6^{\prime \prime} \mathrm{N}, 076^{\circ} 05^{\prime} 06,1^{\prime \prime} \mathrm{O}\right) \mathrm{de}$ la vereda Guanacas, ubicado al lado oeste del Rio Ullucos, a $1.743 \mathrm{msnm}$, con cultivo de pitaya amarilla en pendiente de $15 \%$ y tutorado vivo de maderables en asocio con yuca (Manihot esculenta Crantz, 1766). 3) Predio La Cabaña $\left(02^{\circ} 31^{\prime} 16,6^{\prime \prime} \mathrm{N}, 7^{\circ} 05^{\prime} 52,1^{\prime \prime} \mathrm{O}\right)$ surcado al sureste por el río Ovejas, se encuentra localizado en la vereda Mesopotamia a $1.859 \mathrm{msnm}$. El cultivo de pitaya amarilla se estableció
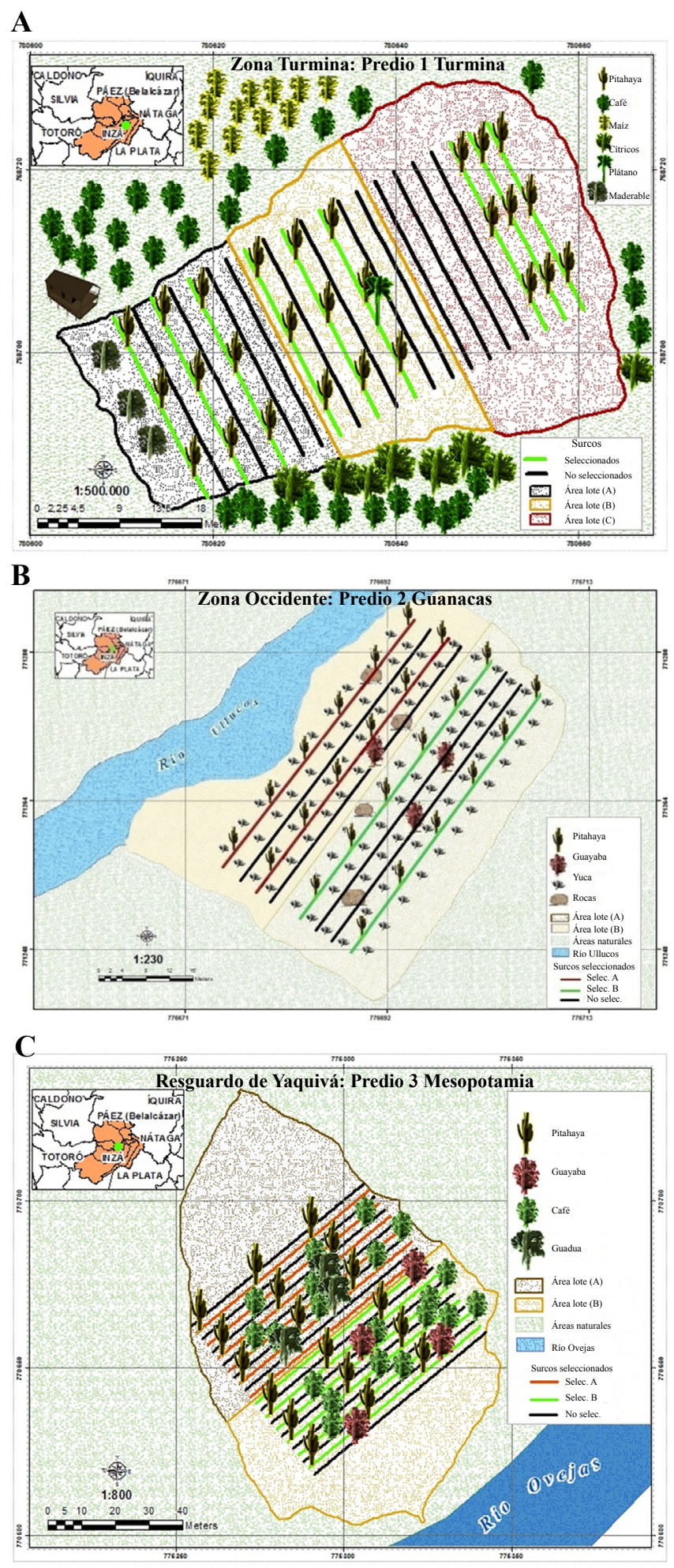

Figura 1. Descripción del área de estudio. A. Predio 1: Piedra Mesa, Vereda Turminá. B. Predio 2: Buena Vista, Vereda Guanacas. C. Predio 3: La Cabaña, Vereda Mesopotamia. Fuente: González et al. 2018.

en terreno con pendiente de $25 \%$ y con tutorado vivo de árboles maderables de flor amarillo y alambre, en asocio con plantas de café (Coffea arabica L., 1753), guayaba (Psidium guajava L., 1753) y guadua (Guadua angustifolia Kunth, 1822) (Fig. 1). La densidad de siembra para el cultivo de pitaya amarilla es de 1,5 m entre plantas por 3,0 m entre surcos en cada predio. El manejo agronómico se realiza 
experimentalmente, con control de arvenses manual y cosecha de frutos teniendo en cuenta el grado de madurez determinado por el color (Martínez et al. 2013). Durante el estudio, se observó permanente floración y fructificación en las plantas y no se realizaron medidas de control fitosanitario a los cultivos.

Componente entomológico. Se realizó un estudio de tipo transversal con tres eventos de muestreo asociados al régimen de lluvias del municipio, así: junio 2016 (100-150 $\mathrm{mm})$, septiembre $2016(100-150 \mathrm{~mm})$ y enero $2017(50-$ $100 \mathrm{~mm}$ ) (rangos de precipitación obtenidas de estaciones climáticas del IDEAM, próximas a los predios evaluados). Los muestreos se realizaron por bloques definidos de acuerdo a la pendiente de cada predio, considerando un tamaño de muestra del $5 \%$ por predio. El predio Piedra Mesa estuvo compuesto por 3 bloques y 27 unidades muestrales, el predio Buena Vista por 2 bloques con 24 unidades muestrales y el predio La Cabaña por 2 bloques con 36 unidades muestrales. Cada planta constituyó una unidad muestral y los muestreos se realizaron entre las 09:00 y 12:00 horas. Se consideraron variables fenológicas como longitud del cladodio más largo de la planta, número de cladodios, número de botones florales y número de frutos.

Los insectos se recolectaron empleando dos métodos realizados de forma simultánea por cultivo: el primero consistió en aspiración bucal y recolección manual directa de los insectos sobre órganos vegetales en todo el componente epigeo de la planta. En el segundo método se realizaron pases dobles con red entomológica a $0,50 \mathrm{~m}$ del tallo de la planta bajo el área foliar y $0,50 \mathrm{~m}$ del suelo. El material entomológico se preservó en alcohol al $70 \%$, en viales individuales etiquetados de acuerdo a la ubicación de la planta en el cultivo. La determinación taxonómica de artrópodos se realizó con base en caracteres morfológicos externos según claves taxonómicas (Sharp 1889; Linnavuori 1959; Korytkowski y Ojeda 1971; González y Carrejo 1992; Norrbom y McAlpine 1996; Delvare et al. 2002; Habeck 2002; Korytkowski 2003; Resh y Cardé 2003; Triplehorn y Johnson 2005; Dietrich 2005; Brown et al. 2009; Jones y Deitz 2009; Thyssen 2010; Hernández 2013; Fernandes et al. 2015). La determinación de individuos de la familia Lonchaeidae (Diptera) se realizó con base en caracteres morfológicos (Castro 2012) y confirmación con experto.

Para cada grupo insectil encontrado se determinó la abundancia relativa y su asociación con las variables fenológicas de la planta. Se calcularon los índices ecológicos de riqueza específica de especies (Margalef, $\mathrm{D}_{\mathrm{Mg}}$ ), de diversidad (Shannon-Wiener, H') y de recambio de especies (Whittaker, $\beta \mathrm{w}$ ). Los resultados obtenidos en campo se sometieron a un análisis descriptivo y de varianza con un nivel de significancia de $\mathrm{P}<0,05$ con comprobación de los supuestos de homogeneidad (prueba de Levene) y normalidad (prueba Kolmogorov-Smirnov). Las variables que no se ajustaron a la distribución normal se analizaron mediante pruebas no paramétricas (Test de Kruskal-Wallis y Kendall) con un nivel de significancia $\mathrm{P}<0,05$. La relación entre la composición de la entomofauna, variables climáticas y fenológicas de las plantas se estimaron mediante correlación de Kendall (Calderón y Alzamora 2011). Todos los análisis estadísticos se realizaron con SPSS $22^{\circ}$ (IBM Corp. Released 2013) siguiendo los métodos propuestos por Guisandé et al. (2013).

\section{Resultados}

En el muestreo directo de insectos asociado a cada órgano y estado fenológico de las plantas, en todos los predios y eventos de muestreo, se recolectó un total de 170 individuos representados por 9 órdenes y 58 familias (Tabla 1). En ningún caso se observaron daños en las plantas atribuibles a especies registradas como limitantes al cultivo (Kondo et al. 2013a).

La familia Lonchaeidae (Diptera) estuvo representada por tres individuos del género Lonchaea y cuatro de Dasiops. Todos correspondieron a machos, lo que imposibilitó la determinación a especie, debido a que se requiere el análisis de la forma del ovipositor (Castro 2012). Lonchaea se reconoció por la presencia de lúnula pequeña claramente pilosa con cerdas en el margen superior, antenas casi juntas en la base con un tercer segmento antenal alargado y de forma ovalada que sobrepasa levemente el margen oral y una cerda esternopleural. Dasiops se reconoció por presentar una lúnula amplia pubescente, base de las antenas ampliamente separada y un tercer segmento antenal corto y redondeado (Korytkowski y Ojeda 1971). Las hormigas recolectadas por el método 1 se hallaron en los cladodios, frutos y botones en todos los predios. Se determinaron como individuos del género Crematogaster spp., al presentar lóbulos frontales bien desarrollados y expandidos, glándula metatibial ausente; espiráculos de los segmentos dos a cuatro del gáster no expuestos, escondidos bajo el tergo de los segmentos anteriores (Palacio y Fernández 2003; Fernández y Sharkey 2006). La familia Coreidae (Hemiptera) estuvo representada por estados inmaduros asociados siempre a cladodios de la zona media de las plantas. Su determinación taxonómica se basó en la ausencia de tricobotrias en la cabeza pero presentes ventralmente en el abdomen, primordios de las alas mesotorácicas cercanos entre sí, lóbulo escutelar sin porción libre, glándulas abdominales levemente separadas posteriormente, tergum $V$ no constreñido en la línea media, antenas con el primer segmento antenal más corto que la longitud conjunta entre el tórax y la cabeza; y en vista lateral, la antena se inserta por encima de la línea entre el centro del ojo y el ápice del tylus (Herring y Ashlock 1971). Posiblemente los especímenes corresponden a la subfamilia Coreinae al presentar coxas cercanas entre si, ausencia de

Tabla 1. Familias de insectos encontrados en predios productivos para pitaya amarilla, Inzá, Cauca 2016-2017.

\begin{tabular}{clccc}
\hline \multirow{2}{*}{ Orden } & \multicolumn{1}{c}{ Familia } & $\begin{array}{c}\text { Piedra } \\
\text { Mesa (n) }\end{array}$ & $\begin{array}{c}\text { Buena } \\
\text { Vista (n) }\end{array}$ & $\begin{array}{c}\text { La Cabaña } \\
\text { (n) }\end{array}$ \\
\hline \multirow{3}{*}{ Diptera } & Syrphidae & - & $\mathrm{Cb}, \mathrm{Cm},(4)$ & $\mathrm{Cm}, \mathrm{B}(3)$ \\
& Lonchaeidae & $\mathrm{Ca}, \mathrm{Cm}, \mathrm{F}(4)$ & $\mathrm{F} \mathrm{(1)}$ & - \\
& Tachinidae & $\mathrm{Cm}(1)$ & - & $\mathrm{Cm}, \mathrm{B}(2)$ \\
\hline \multirow{4}{*}{ Hemiptera } & Reduviidae & - & $\mathrm{Cb}(1)$ & - \\
& Miridae & - & $\mathrm{Cm}(1)$ & $\mathrm{Cm}(2)$ \\
& Coreidae & $\mathrm{Cm}(10)$ & - & $\mathrm{Cm}(1)$ \\
\hline \multirow{2}{*}{ Coleoptera } & Chrysomelidae & $\mathrm{Cm}(2)$ & & $\mathrm{Ca}, \mathrm{Cm}(4)$ \\
& Coccinellidae & $\mathrm{Ca}(1)$ & $\mathrm{Cm}(3)$ & \\
\hline \multirow{4}{*}{ Hymenoptera } & Apidae & $\mathrm{B}, \mathrm{F}(2)$ & - & $\mathrm{B} \mathrm{(1)}$ \\
& Fraconidae & $\mathrm{Cm}(3)$ & - & \\
& Ichneumonidae & - & $\mathrm{Pa}, \mathrm{F}(2)$ & - \\
\hline
\end{tabular}

Ca: Cladodio zona alta. $\mathrm{Cm}$ : Cladodio zona media. $\mathrm{Cb}$ : Cladodio zona baja. $\mathrm{F}$ : Fruto. B: Botón. 
glándula metatorácica, presencia de estrías en el fémur y ausencia de espinas en la tibia (Fernandes et al. 2015).

No se encontraron diferencias estadísticamente significativas en la distribución espacial de los individuos entre los bloques o entre la abundancia relativa de insectos para todos los eventos de muestreo en los predios evaluados (Tabla 2). Sin embargo, para el predio Piedra Mesa la mayor abundancia relativa de insectos se obtuvo en cladodios medios y altos para todos los eventos de muestreo, mientras que solo hubo asociación de individuos con flores y frutos en los muestreos 2 y 3 . En el predio Buena Vista, la mayor cantidad de individuos se encontró en cladodios altos, medios y bajos y no se encontraron individuos en botones y en flores. En el predio La Cabaña, la fauna insectil se encontró asociada a cladodios altos, medios y bajos de las plantas (Figs. 2A-2C). Sin embargo, se encontró correlación positiva entre plantas con mayor densidad de cladodios y el número de individuos la familia Lonchaeidae $(\tau=1,0 ; \mathrm{P}=0,00)$ y estos con las variables 'temperatura' $(\tau=1,0 ; \mathrm{P}=0,00)$ y 'precipitación' $(\tau=$ $1,0 ; \mathrm{P}=0,00)$. En el predio Buena Vista, las poblaciones de las familias Formicidae y Syrphidae presentaron una correlación positiva con la 'humedad relativa' $(\tau=1,00 ; \mathrm{P}=0,007)$. El comportamiento de la familia Lonchaeidae en el predio La Cabaña presentó correlación positiva con la variable número de frutos $(\tau=1,0 ; \mathrm{P}=0,00)$.

La biodiversidad basada en insectos en los predios evaluados reveló una riqueza equiparable con sistemas de baja intervención antrópica (Piedra Mesa $\mathrm{D}_{\mathrm{Mg}}=7,01$; Buena vista $\mathrm{D}_{\mathrm{Mg}}=6,46$; La Cabaña $\left.\mathrm{D}_{\mathrm{Mg}}=7,42\right)$, siendo ligeramente menor en el predio Buena Vista, con menor diversidad de plantas y a menor altitud. Entre predios se encontró alta heterogeneidad con diversidad moderada de insectos, ligeramente mayor en el predio de La Cabaña con menor intensidad de prácticas agronómicas (Índices de Shannon: Piedra Mesa H' = 3,089; Buena Vista $H^{\prime}=3,005$; La Cabaña $\left.H^{\prime}=3,217\right)$. En general, se encontró homogeneidad para la zona de estudio $(\beta \mathrm{w}=1,0)$ con bajo solape de familias de insectos $(\beta r=0,25)$.

El muestreo realizado con el método 2 permitió registrar a Lepidoptera, no encontrado con el método 1 y en total, 56 familias de insectos asociadas a los cultivos de pitaya amarilla en los predios productivos evaluados: Buena Vista $n=69, \mathrm{La}$ Cabaña $n=63$, Piedra Mesa $n=58$ (Tabla 3).

\section{Discusión}

La pitaya amarilla constituye un cultivo introducido en Inzá-Cauca, que por su carácter perenne puede modificar de forma importante la dinámica poblacional y las interacciones ecológicas de las poblaciones de insectos, favoreciendo aquellas que puedan obtener mayor beneficio del nuevo recurso (Mack et al. 2000). La producción en pequeñas

Tabla 2. Comparación de la distribución espacial y abundancia relativa de insectos entre muestreos para los predios evaluados $P>0,05$. Test de Kruskal-Wallis y Kendall.

\begin{tabular}{ccccc}
\hline \multicolumn{1}{c}{ Predio } & M1 & Mloques & M3 & $\begin{array}{c}\text { Eventos de } \\
\text { muestreo }\end{array}$ \\
\hline Piedra Mesa & $\mathrm{P}=0,278$ & $\mathrm{P}=0,721$ & $\mathrm{P}=0,897$ & $\mathrm{P}=0,463$ \\
Buena Vista & $\mathrm{P}=0,023$ & $\mathrm{P}=0,889$ & $\mathrm{P}=0,340$ & $\mathrm{P}=0,028$ \\
La Cabaña & $\mathrm{P}=0,380$ & $\mathrm{P}=0,224$ & $\mathrm{P}=0,107$ & $\mathrm{P}=0,760$ \\
\hline
\end{tabular}

$\mathrm{M} 1=$ muestreo $1, \mathrm{M} 2=$ muestreo $2, \mathrm{M} 3=$ muestreo. parcelas a modo de policultivos, con labranza mínima y un manejo fitosanitario basado en podas y remoción manual de arvenses en calles y plato, genera menor impacto en la biodiversidad insectil permitiendo la representatividad de familias de insectos benéficos para el sistema productivo, lo que promueve relaciones tróficas con tendencia al equilibrio de las poblaciones y sus distintas funciones en el
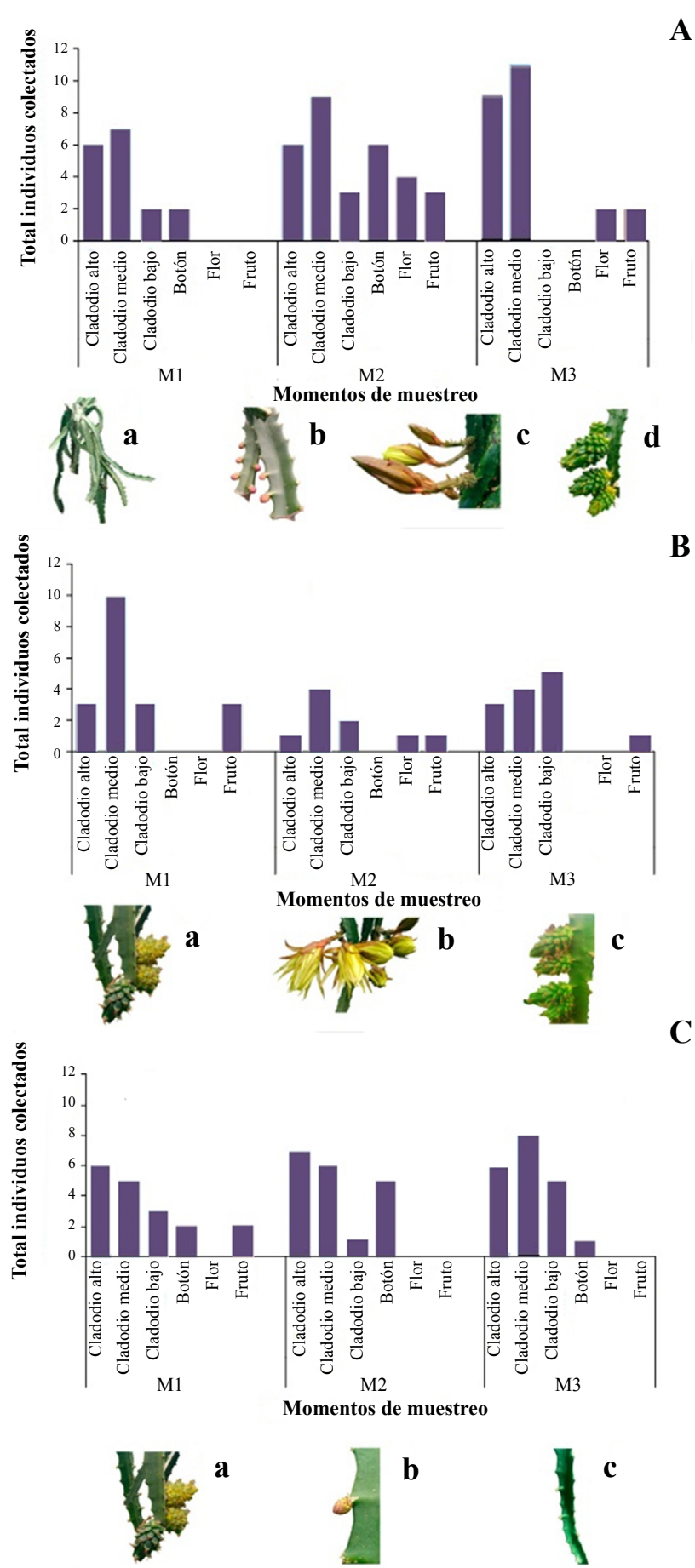

Figura 2. Población total $(n=170)$ total de insectos recolectados en los órganos vegetales de pitaya amarilla (Selenicereus megalanthus) por evento de muestreo (M1, M2 y M3) en el municipio de Inzá, Cauca. M1 (100-150 mm), M2 (100-150 mm) y M3 (50-100 mm). A. Predio 1: Piedra Mesa, Vereda Turminá. B. Predio 2: Buena Vista, Vereda Guanacas. C. Predio 3: La Cabaña, Mesopotamia. Fenología de la planta encontrada para cada evento de muestreo: a. Cladodios. b. Botones. c. Flores. d. Frutos. Fuente: González et al. 2018. 
Tabla 3. Familias de insectos encontrados mediante el método 2 en predios productivos de pitaya amarilla, Inzá, Cauca 2016-2017. n = número de individuos.

\begin{tabular}{lccc}
\hline \multicolumn{1}{c}{$\begin{array}{c}\text { Orden / Familia / } \\
\text { Subfamilia/ Género }\end{array}$} & $\begin{array}{c}\text { Buena Vista } \\
\text { (n) }\end{array}$ & $\begin{array}{c}\text { La Cabaña } \\
\text { (n) }\end{array}$ & $\begin{array}{c}\text { Piedra Mesa } \\
\text { (n) }\end{array}$ \\
\hline COLEOPTERA & & & \\
Carabidae & & 2 & 2 \\
Chrysomelidae & 1 & & 1 \\
Curculionidae & 1 & 1 & 2 \\
Dytiscidae & & & 1 \\
Hybosoridae & & & 1 \\
Lycidae & & & 1 \\
Nitidulidae & & & 10 \\
$\quad$ Carpophius sp. & 1 & & \\
$\quad$ Glischrochilus sp. & & & \\
Staphylinidae & 1 & 2 &
\end{tabular}

\section{DIPTERA}

\begin{tabular}{|c|c|c|c|}
\hline Agromyzidae & 5 & & \\
\hline Anthomyzidae & 3 & 1 & \\
\hline Asteiidae & & & 10 \\
\hline Cecidomyiidae & 1 & & \\
\hline Ceratopogonidae & & 1 & 1 \\
\hline Chloropidae & 2 & 3 & \\
\hline Cryptochetidae & & 1 & \\
\hline Dixidae & 1 & & \\
\hline Dolichopodidae & 1 & 4 & 2 \\
\hline Drosophilidae & 6 & & 2 \\
\hline Empididae & & 1 & \\
\hline Heleomyzidae & 1 & & \\
\hline Lonchaeidae & 2 & & \\
\hline Milichiidae & & & 7 \\
\hline Mycetophilidae & & 1 & \\
\hline Phoridae & & & 8 \\
\hline Sciaridae & & 1 & 6 \\
\hline Somatiidae & 1 & & \\
\hline Sphaeroceridae & 5 & & 1 \\
\hline Syrphidae & 9 & 3 & \\
\hline Tipulidae & 1 & 1 & \\
\hline
\end{tabular}

\section{HEMIPTERA}

Alydidae

Aphididae

Aradidae

sistema (Harvey et al. 2004; Schoonhoven 2005; Weyland y Zaccagnini 2008; Altieri y Nicholls 2007; Gutiérrez et al. 2007). No obstante, debido a que se encontró una correlación positiva entre variables abióticas y algunos grupos de insectos asociados al número de cladodios y el número de frutos de pitaya amarilla en los predios evaluados, debe enfatizarse en la arquitectura de las plantas durante su establecimiento, con miras a la regulación del microclima al interior de los cultivos (Pérez 1999; Medina y Kondo 2012; García 2016; Torres y García 2014; Kolb et al. 2016) como estrategia de

\begin{tabular}{|c|c|c|c|}
\hline $\begin{array}{c}\text { Orden / Familia / } \\
\text { Subfamilia/ Género }\end{array}$ & $\begin{array}{l}\text { Buena Vista } \\
\text { (n) }\end{array}$ & $\begin{array}{l}\text { La Cabaña } \\
\text { (n) }\end{array}$ & $\begin{array}{l}\text { Piedra Mesa } \\
\text { (n) }\end{array}$ \\
\hline Berytidae & 1 & & \\
\hline Cercopidae & & 1 & \\
\hline \multicolumn{4}{|l|}{ Cicadellidae } \\
\hline Cicadellinae & 1 & & \\
\hline Deltocephalinae & 1 & & \\
\hline Megophthalminae & & 2 & \\
\hline \multicolumn{4}{|l|}{ Ledrinae } \\
\hline Xerophloea sp. & & 1 & \\
\hline Coreidae & & 1 & \\
\hline Cydnidae & 3 & 6 & \\
\hline Geocoridae & & & 1 \\
\hline Lygaeidae & & 4 & 1 \\
\hline Miridae & 1 & & \\
\hline Pachygrontidae & & 1 & \\
\hline Pentatomidae & 1 & & \\
\hline Reduviidae & 2 & 1 & \\
\hline Rhopalidae & & 2 & \\
\hline Rhyparochromidae & 1 & 4 & \\
\hline Thyreocoridae & & 3 & \\
\hline \multicolumn{4}{|l|}{ HYMENOPTERA } \\
\hline Apidae & & 1 & \\
\hline \multicolumn{4}{|l|}{ Braconidae } \\
\hline Braconinae & 1 & & \\
\hline Opiinae & 1 & & \\
\hline \multicolumn{4}{|l|}{ Formicidae } \\
\hline Acromyrmex sp. & 6 & 2 & \\
\hline \multicolumn{4}{|l|}{ Ichneumonidae } \\
\hline Cryptinae & 1 & & \\
\hline Pteromalidae & & 1 & \\
\hline Sphecidae & 1 & & \\
\hline \multicolumn{4}{|l|}{ LEPIDOPTERA } \\
\hline Geometridae & & 1 & \\
\hline \multicolumn{4}{|l|}{ ORTHOPTERA } \\
\hline Acrididae & 4 & 7 & \\
\hline Gryllidae & & 1 & \\
\hline Tettigoniidae & & 1 & \\
\hline Total & 69 & 63 & 58 \\
\hline
\end{tabular}

...continuación Tabla 3

prevención y manejo fitosanitario, particularmente en épocas de alta precipitación.

Dasiops saltans puede llegar a ocasionar pérdidas entre 40-80 \% del rendimiento del cultivo de pitaya amarilla en un ciclo de floración, debido a que las larvas consumen el interior del botón floral (Vergara y Pérez 1988; Delgado et al. 2010). Aunque se encontraron especímenes del género Dasiops spp. en los predios con pitaya amarilla en Inzá, se sabe que el género está representado por lo menos por 45 especies asociadas a múltiples hospedantes (MacGowan 2017), por lo 
que no puede concluirse la presencia de $D$. saltans para esta zona de estudio.

No se encontró clorosis o punturas por daño asociado con las chinches del género Leptoglossus, pero las poblaciones deben ser vigiladas porque formas inmaduras de la familia Coreidae se asociaron con cladodios de la zona media de las plantas, como ha sido registrado para L. zonatus en otras zonas productoras de pitaya amarilla del país (Medina y Kondo 2012). A pesar de los hábitos polífagos de esta especie (Kondo et al. 2013a; Medina y Kondo 2012) y a la heterogeneidad del arreglo de los cultivos de pitaya en Inzá, debe realizarse seguimiento a las poblaciones del insecto, no solo por los daños directos ocasionados por sus hábitos de alimentación sino por el oportunismo que genera para la colonización de patógenos en la planta (Kondo et al. 2013a).

El país cuenta con información que permite el reconocimiento temprano de daños asociados a insectos en pitaya amarilla (Kondo et al. 2013a) por lo que en aras de mantener el equilibrio trófico en las poblaciones de artrópodos que redunde en la producción limpia de pitaya amarilla en Inzá, se requiere capacitación a los productores para el reconocimiento de las principales plagas. Por ejemplo, además de $D$. saltans, plaga clave, se ha registrado a Lonchaea longicornis Willinston, 1896 y Neosilba batesi (Curran, 1932) (Medina y Kondo 2012), de las cuales no se tiene definida su asociación con el cultivo. Aunque los tres géneros, que pertenecen a la familia Lonchaeidae no se diferencian a simple vista, la asociación con el daño en frutos, permitiría un sistema de alerta temprana para la mosca del botón floral de pitaya amarilla. Existe un importante riesgo de introducción y establecimiento de $D$. saltans en Inzá, proceso que puede favorecerse a través de transporte pasivo por los corredores de comercialización con zonas productoras de pitaya amarilla cercanas a Inzá en los departamentos del Huila y Valle del Cauca, donde la especie constituye el principal problema fitosanitario (Kondo et al. 2009; Delgado et al. 2010) y por las características ecológicas de la zona que pueden constituir un nicho apropiado para esta especie limitante (Patiño-Tira et al. 2013).

Los índices ecológicos revelaron una alta riqueza y diversidad de grupos insectiles, muy posiblemente por la contribución de familias con funciones benéficas como parasitoides (Braconidae e Ichneumonidae), depredadores (Syrphidae y Reduviidae), posibles cleptoparásitos de la familia Milichiidae, que podrían estar relacionadas a la presencia de Leptoglossus zonatus (Dallas, 1852) (Kondo et al. 2010) y polinizadores (Syrphidae, Nitidulidae y Apidae), particularmente en los predios Buena Vista y La Cabaña, caracterizados por un manejo agronómico no intensivo y alta diversidad vegetal presente con distintos cultivos acompañantes como yuca y maderables en el primer predio y con café, guayaba y guadua en el segundo. La promoción de prácticas agronómicas compatibles con la preservación de esta biodiversidad debe ser vigilada para evitar el aumento en la dominancia y consecuente reducción de la equitabilidad de grupos y desbalance trófico.

A pesar de no contar con criterios técnicos para el manejo de los cultivos comerciales de pitaya amarilla en Inzá, los agricultores establecieron una asociación para su comercialización nacional e internacional, por lo que se espera un negocio de largo aliento en la zona. Durante el desarrollo del estudio, los frutos presentaron sanidad fitosanitaria, excelentes cualidades organolépticas y gran tamaño (con peso promedio de $433 \mathrm{~g}$ ). Debido a los retrasos en el desarrollo fenológico de las plantas, definido por estar localizados en zona marginal para el cultivo (Alarcón et al. 1996; García y Straube 1998; Wintgens 2004; AlvaradoGaona et al. 2015), la cosecha está desfasada con respecto a la producción nacional, lo que favorece su comercialización. Aunado al impacto familiar, económico y social que ha tenido el cultivo de pitaya amarilla en Inzá-Cauca, se espera la ampliación de hectáreas sembradas, por lo que la información que aporte a la vigilancia entomológica es fundamental para un manejo integrado de plagas basado en criterios técnicos y de evaluación.

Colombia cuenta con importante información sobre el cultivo de pitaya amarilla (Ayala et al. 2009; Jiménez et al. 2012; Kondo et al. 2013b), por lo que debe establecerse un sistema de acompañamiento a productores que permita la adopción de sistemas de vigilancia fitosanitaria y de estrategias de manejo agronómico con criterio técnico, amigables con el ambiente y con perspectiva a largo plazo. La evaluación permanente de los sistemas productivos en InzáCauca cobra especial importancia para alimentar los sistemas de alerta temprana para enfermedades y plagas, ya que una vez establecidos pueden generar pérdidas muy importantes. El equilibrio de las relaciones planta-insecto que se describen en el presente estudio deberían mantenerse a largo plazo, para lo cual la vigilancia entomológica regular es esencial.

\section{Conclusiones}

Las características del sistema de siembra tipo policultivo a pequeña escala para pitaya amarilla en Inzá, Cauca, fomentan la riqueza y diversidad de insectos con amplia representación de benéficos, base fundamental de relaciones tróficas complejas en beneficio del equilibrio del ecosistema. Aunque los hallazgos del estudio no revelaron la existencia de D. saltans y L. zonatus, descritas como insectos limitantes para pitaya amarilla para el país, se deben implementar sistemas de alerta temprana para reducir las posibilidades de ingreso y establecimiento de poblaciones de estas especies en la zona. Esta estrategia es fundamental para fortalecer el posicionamiento del municipio de Inzá como productor y exportador de la fruta, particularmente en épocas de baja producción en otras zonas del país.

\section{Agradecimientos}

A los productores de pitahaya amarilla del municipio de Inzá Cauca, a Heidy Xiomara Ortega Microbióloga MSc, Angela Patricia Castro Avila MSc, y al Semillero de Entomología del laboratorio de Entomología de la Facultad de Ciencias Agrarias de la Universidad Nacional de Colombia, sede Bogotá.

\section{Literatura citada}

ALARCÓN, M.; ALDAZABAL, O.; MARTÍNEZ, J. 1996. Influencia del sol y la sombra en la calidad y el rendimiento del grano de café. Centro Agrícola 23 (3): 11-16.

ALTIERI, M.; NICHOLLS, C. 2007. Biodiversidad y manejo de plagas en agroecosistemas. Icaria Editorial. Barcelona, España $248 \mathrm{p}$.

ALVARADO-GAONA, A.; CASTELLANOS, E.; FONSECA, L. 2015. Sistema productivo del cultivo de pitaya amarilla 
(Selenicereus megalanthus) en Boyacá-Colombia. Revista Innovación y Desarrollo 4 (9): 146-161. https://doi.org/10.31644/ IMASD.9.2015.a07

AYALA, A.; SERNA-COCK, L.; GIRALDO, C. 2009. Efecto de la agitación sobre la deshidratación osmótica de pitahaya amarilla (Selenicereus megalanthus S.) empleando soluciones de sacarosa. Interciencia (Venezuela) 34 (7): 492-496.

BARTHLOTT, W.; HUNT, D. 1993. Cactaceae. pp. 161-197. En: Kubitzki, K.; Rohwer, J. G.; Bittrich, V. (Eds.). Flowering Plants. Dicotyledons. The families and genera of vascular plants. Vol II. Springer, Berlin, Heidelberg 653 p. https://doi.org/10.1007/9783-662-02899-5_17

BETANCOURT, G.; TORO, J.; MOSQUERA, A. 2010. Agenda prospectiva de investigación y desarrollo tecnológico para la cadena productiva de la pitaya amarilla en fresco en el Valle del Cauca. Ministerio de Agricultura y Desarrollo Rural, Universidad del Valle, Universidad Nacional de Colombia, Servicio Nacional de Aprendizaje - SENA, Colciencias, Bogotá 152 p.

BROWN, B.; BORKENT, A.; CUMMING, J.; WOOD, D.; WOODLEY, N.; ZUMBADO, M. 2009. Manual of Central America Diptera. Volume I. Ottawa, NRC Research Press 714 p.

CALDERÓN, J.; ALZAMORA, L. 2011. Estadística para la tesis de postgrado. Lulu international, Safe Creative. Lima, Perú 135 p.

CASTRO, A. 2012. Dasiops Rondani (Diptera: Lonchaeidae) asociadas a pasifloras cultivadas en Colombia. Tesis M. Sc, Facultad de Agronomía, Universidad Nacional de Colombia, Bogotá. Disponible en: http://bdigital.unal.edu. co/7588/1/790724.2012.pdf [Fecha revisión: enero 2018].

DELGADO, A.; KONDO, T.; IMBACHI, K.; QUINTERO, E.; MANRIQUE, M.; MEDINA, J. 2010. Biología y algunos datos morfológicos de la mosca del botón floral de la pitahaya amarilla, Dasiops saltans (Townsend) (Diptera: Lonchaeidae) en el Valle del Cauca. Boletín del Museo Entomológico de la Universidad del Valle 11 (2): 1-10.

DELVARE, G.; ABERLENC, H.; MICHEL, B.; FIGUEROA, A. 2002. Los insectos de África y de América tropical claves para la identificación de las principales familias, Ed 1. CIRAD Montpellier, Francia, 102-259 p.

DIETRICH, C. H. 2005. Keys to the families of Cicadomorpha and subfamilies and tribes of Cicadellidae (Hemiptera: Auchenorrhyncha). Florida Entomologist 88 (4): 502-517. https:// doi.org/10.1653/0015-4040(2005)88[502:KTTFOC]2.0.CO;2

FERNANDES, J.; LEVIN, P.; LIVERMORE, L.; NIKUNLASSI, M. 2015. Leaf-Footed bugs (Coreidae). pp. 549-605. En: Panizzi, A.; Grazia, J. (Eds.). True bugs (Heteroptera) of the Neotropics. Springer Science Business Media Dordrecht, Entomology in Focus 2, 557 p. https://doi.org/10.1007/978-94-017-9861-7_19

FERNÁNDEZ, F.; SHARKEY, M. 2006. Introducción a los Hymenoptera de la Región Neotropical. Sociedad Colombiana de Entomología y Universidad Nacional de Colombia. Bogotá, D. C. 894 p.

GARCÍA, F.; STRAUBE, U. 1998. La sombra en el cafetal. Manual de caficultura. Anacafe. Ciudad de Guatemala, Guatemala 81-98.

GARCÍA, J. 2016. Fenología y herbívoros plaga del cultivo de curuba en Pasca, Cundinamarca. Tesis M. Sc., Facultad de Agronomía, Universidad Nacional de Colombia, Bogotá. Disponible en: http:// bdigital.unal.edu.co/52359/1/jennylilianagarciamorantes.2016. pdf [Fecha revisión: enero 2018].

GOBERNACIÓN DEL CAUCA. 2013. Línea base de indicadores económicos, Diagnóstico de condiciones sociales y económicas, Municipio de Inzá. 162 p.

GONZÁLEZ, R.; CARREJO, N. 1992. Introducción al estudio de los Diptera. Centro Editorial Universidad de Valle. Cali, Colombia $79 \mathrm{p}$.

GONZÁLEZ, T.; PERAZA, A.; SILVA, A.; TOMBE, L.; MORENO, E.; ORTIZ, A.; SANTAMARIA, M.; BROCHERO, H. 2018. Insectos presentes en cultivos de Pitaya Amarilla, Selenicereus megalanthus (K Schumann ex Vaupel) Moran en el Municipio de Inzá, Cauca, Colombia. Universidad Nacional de Colombia, $56 \mathrm{p}$.
GUISANDÉ, C.; VAAMONDE, A.; BARREIRO, A. 2013. Tratamiento de datos con R, Statistica y SPSS 22. Ed. Díaz de Santos, España 978 p.

GUTIÉRREZ, A.; AGUILAR, C.; GALDÁMEZ, J.; MENDOZA, S. 2007. Importancia ecológica y socioeconómica de los sistemas de policultivos de maíz-fríjol-calabaza en la Frailesca, Chiapas, México. Sociedad Mexicana de Agricultura Sostenible $7 \mathrm{p}$.

HABECK, D. H. 2002. Nitidulidae. pp. 311-315. En: Arnett, R. H; Thomas, M. C.; Skelley, P. E.; Frank, H. J. (Eds.). American beetles. Volume 2. Polyphaga: Scarabaeoidea through Curculionoidea. CRC Press, Boca Raton, $861 \mathrm{p}$.

HARVEY, C.; SÁENZ, J.; MONTERO, J.; MEDINA, A.; SÁNCHEZ, D.; VÍLCHEZ, S.; HERNÁNDEZ, B.; MAES, J.; SINCLAIR, F. 2004. Abundance and species richness of trees, birds, bats, butterflies and dung beetles in a silvopastoral system in the agricultural landscapes of Costa Rica and Nicaragua. Semana Científica, CATIE (6): 82-84.

HERNÁNDEZ, H. 2013. Escarabajos de la savia (Coleoptera: Nitidulidae) de Coahuila, México. Tesis de Maestría en Ciencias en Parasitología Agrícola. Universidad Autónoma Agraria Antonio Narro. Coahuila, México 102 p. Disponible en: http://repositorio. uaaan.mx:8080/xmlui/bitstream/handle/123456789/7090/ Hern $\%$ C $3 \%$ A 1 ndez $\% 20$ Torres $\% 2$ C $\% 20$ Hermelindo $\% 20 \% 20$ Tesis $\% 20$ Maestr $\%$ C3\%ADa.pdf? sequence $=3$ \&isAllowed $=y$ [Fecha revisión: enero 2018].

HERRING, J.; ASHLOCK, P. 1971. A key to the nymphs of the families of Hemiptera (Heteroptera) of America north of Mexico. The Florida Entomologist 54 (3): 207-212. https://doi. org/10.2307/3493715

IBM Corp. Released. 2013. IBM SPSS Statistics for Windows, Version 22.0, Armonk, NY, IBM Corp.

JIMÉNEZ, Y.; CARRANZA, C.; RODRÍGUEZ, M. 2012. Gulupa (Passiflora edulis Sims). pp. 579-599. En: Fischer, G. (Ed.). Manual para el cultivo de frutales en el trópico. Produmedios, Bogotá 1023 p.

JONES, J.; DEITZ, L. 2009. Phylogeny and systematics of the leafhopper subfamily Ledrinae (Hemiptera: Cicadellidae). Zootaxa 2186: 1-120. http://dx.doi.org/10.11646/zootaxa.2186.1.1

KOLB, T.; FETTIG, C.; AYRES, M.; BENTZ, B.; HICKE, J.; MATHIASEN, R.; STEWARTF, J. E.; WEED, A. S. 2016. Observed and anticipated impacts of drought on forest insects and diseases in the United States. Forest Ecology and Management 380: 321-334. https://doi.org/10.1016/j.foreco.2016.04.051

KONDO, T.; IMBACHI, K.; QUINTERO, E.; DELGADO, A.; MANRIQUE, M.; MURILLAS, M.; VÉLEZ, C. 2009. Biología y hábitos de la mosca del botón floral de la pitaya amarilla, Dasiops saltans (Townsend) en el Valle del Cauca, Colombia. Corporación Colombiana de Investigación Agropecuaria, Corpoica. Palmira, Valle del Cauca, Colombia $22 \mathrm{p}$.

KONDO, T.; BRAKE, I.; IMBACHI, K.; KORYTKOWSKI, C. 2010. Report of Milichiella lacteipennis (Diptera: Milichiidae) attracted to various crushed bugs (Hemiptera: Coreidae \& Pentatomidae). Boletín del Museo de Entomología de la Universidad del Valle 11 (1): 16-20.

KONDO, T.; QUINTERO, E.; MEDINA, J.; LÓPEZ, K.; DELGADO, A.; MANRIQUE, M. 2013a. Insectos plagas de importancia económica en el cultivo de pitaya amarilla. pp. 6477. En: Kondo, T.; Martínez, M.; Medina, J.; Rebolledo-Roa, A.; Cardozo-Burgos, C. (Eds.). Manual técnico: Tecnología para el manejo de pitaya amarilla Selenicereus megalanthus (K. Schum. Ex Vaupel) Moran en Colombia. Corporación Colombiana de Investigación Agropecuaria, Corpoica. Palmira, Valle del Cauca, Colombia. $96 \mathrm{p}$.

KONDO, T.; MARTÍNEZ, M.; MEDINA, J. A.; REBOLLEDOROA, A.; CARDOZO-BURGOS, C. (Eds.). 2013b. Manual Técnico: Tecnología para el manejo de pitaya amarilla Selenicereus megalanthus (K. Schum. ex Vaupel) Moran en Colombia. Corporación Colombiana de Investigación Agropecuaria, Corpoica. Palmira, Valle del Cauca, Colombia. $96 \mathrm{p}$. 
KORYTKOWSKI, C. 2003. Manual de identificación de moscas de la fruta. Parte 1: Generalidades sobre clasificación y evolución de Acalyptratae, familias Neriidae, Ropalomeridae, Lonchaeidae, Richardiidae, Otitidae y Tephritidae. Programa de Maestría en Entomología. Universidad de Panamá. 34 p.

KORYTKOWSKI, C.; OJEDA, D. 1971. Revisión de las especies de la familia Lonchaeidae en el Perú (Diptera: Acalyptratae). Revista Peruana de Entomología 14 (1): 87-116.

MACGOWAN, I. 2017. Lonchaeidae online. Disponible en: http:// lonchaeidae.myspecies.info/ [Fecha revisión: enero 2018].

MACK, R.; SIMBERLOFF, D.; LONSDALE, M.; EVANS, H.; CLOUT, M.; BAZZAZ, F. 2000. Biotic invasions: Causes, epidemiology global consequences and control. Ecological Applications 10 (3): 689-710. https://doi.org/10.1890/10510761(2000)010[0689:BICEGC]2.0.CO;2

MARTÍNEZ, M.; MEDINA, J.; MUÑOZ, D. 2013. Cosecha. pp. 8890. En: Kondo, T.; Martínez, M.; Medina, J.; Rebolledo-Roa, A.; Cardozo-Burgos, C. (Eds.). Manual técnico: Tecnología para el manejo de pitaya amarilla Selenicereus megalanthus (K. Schum. Ex Vaupel) Moran en Colombia. Corporación Colombiana de Investigación Agropecuaria, Corpoica. Palmira, Valle del Cauca, Colombia. $96 \mathrm{p}$.

MEDINA, J.; KONDO, T. 2012. Listado taxonómico de organismos que afectan la pitaya amarilla, Selenicereus megalanthus (K. Schum. ex Vaupel) Moran (Cactaceae) en Colombia. Revista Corporación Colombiana de Investigación Agropecuaria, Corpoica - Ciencia y Tecnología Agropecuaria 13 (1): 41-46. https://doi.org/10.21930/rcta.vol13 num1 art:238

NOBEL, P. 2002. Cacti: Biology and uses. University of California Press. Berkeley and Los Angeles, California, EE. UU. 280 p.

NORRBOM, A.; MCALPINE, J. 1996. A revision of Neotropical species of Dasiops Rondani (Diptera: Lonchaeidae) attacking passiflora (Passifloraceae). Memoirs Entomological Society 18: 189-211.

LINNAVUORI, R. 1959. Revision of the Neotropical Deltocephalinae and some related subfamilies (Homoptera). Societas Zoologica Botanica Fennica "Vanamo" 20: 1-370.

PALACIO, E.; FERNÁNDEZ, F. 2003. Clave para las subfamilias y géneros. pp. 242-251. En: Fernández, F. Introducción a las hormigas de la región Neotropical. Instituto de Investigación de Recursos Biológico Alexander von Humboldt, Bogotá, Colombia. XXVI, 398 p.

PATIÑO-TIRA, H.; MARTÍNEZ, J.; ALVARADO, A. 2013. Inventario de la entomofauna asociada al cultivo de pitahaya amarilla (Selenicereus megalanthus Haw.) en Briceño (Boyacá). Ciencia y Agricultura 11 (1): 67-76. https://doi. org/10.19053/01228420.3489

PÉREZ, T. 1999. La especialización en los insectos fitófagos: Una regla más que una excepción. Boletín de la Sociedad Entomológica Aragonesa 26: 759-776.

REBOLLEDO, A.; DURÁN, A.; LABRADOR, N. 2003. Ecofisiología y comportamiento productivo. pp. 28-39. En: Kondo, T.; Martínez, M.; Medina, J.; Rebolledo-Roa, A.; Cardozo-Burgos, C. (Eds.). Manual técnico: Tecnología para el manejo de pitaya amarilla Selenicereus megalanthus (K. Schum. Ex Vaupel) Moran en Colombia. Corporación Colombiana de Investigación Agropecuaria, Corpoica. Palmira, Valle del Cauca, Colombia. $96 \mathrm{p}$.

RESH, V.; CARDE, R. 2003. The encyclopedia of insects. Academic Press, San Diego 265 p.

RODRÍGUEZ, C. 2002. Pitahaya (Hylocereus undatus y Selenicereus megalanthus). Producción y comercialización en el mundo y en México. pp. 63-95. En: Flores, V. C. (Ed.). Pitayas y pitahayas, producción, poscosecha, industrialización y comercialización. Ed. CIESTAAM-UACH. Chapingo, Edo. de México. Disponible en: http://www.infoaserca.gob.mx/claridades/revistas/082/ ca082.pdf. [Fecha revisión: enero 2018].
SCHOONHOVEN, L.; VAN LOON, J.; DICKE, M. 2005. Insectplant biology. Ed II. Oxford University press $440 \mathrm{p}$.

SHARP, D. 1889. Nitidulidae. pp. 265-388. En: Godman, F.; Osbert, S. (Eds.). Biologia Centrali-Americana Coleoptera 2 (1). Bernard Quaritch Ltd., Londres, Inglaterra 727 p.

TEL-ZUR, N.; ABBO, S.; BAR-ZVI, D.; MIZRAHI, Y. 2004. Clone identification and genetic relationship among wine cacti from the genera Hylocereus and Selenicereus based on RAPD analysis. Scientia Horticulturae 100: 279-289. https://doi.org/10.1016/j. scienta.2003.09.007

THYSSEN, P. 2010. Keys to identification of immature insects. pp. 25-42. En: Amendt, J.; Goff, M. L.; Campobasso, C. P.; Grassberger, M. (Eds.). Current concepts in forensic entomology. New York. Springer. 376 p. https://doi.org/10.1007/978-1-40209684-6 2

TORRES, D.; GARCÍA, G. 2014. Análisis del papel de los caracteres foliares de Cnidoscolus (Euphorbiaceae) en la defensa contra herbívoros y patógenos. Revista Especializada en Ciencias Químico-Biológicas 17: 126-134. https://doi.org/10.1016/ S1405-888X(14)72087-X

TRIPLEHORN, C.; JOHNSON, N. 2005. Study of Insects. 7a Edición. Saunders College Publishing. 888 p.

VERGARA, R.; PÉREZ, D. 1988. Biosíntesis: Plagas del cultivo de la pitahaya. Revista Siatol 2: 30-46.

WEYLAND, F.; ZACCAGNINI, M. 2008. Efecto de las terrazas sobre la diversidad de artrópodos caminadores en cultivos de soja. Instituto de Recursos Biológicos (IRB). Revista Ecología Austral 18: 357-366.

WINTGENS, J. 2004. Factors influencing the quality of green coffee. pp. 798-809. En: Wintgens, J. (Ed.). Coffee: Growing, Processing, Sustainable Production. Alemania, Wiley-VCH. 1022 p. https://doi.org/10.1002/9783527619627

\section{Origen y financiamiento}

El presente documento se origina del trabajo de grado "Caracterización de insectos plaga asociados a cultivos de pitahaya amarilla (Selenicereus megalanthus K. Schum. Ex Vaupel) Moran en el municipio de Inzá, Cauca, Colombia" presentado en la Facultad de Ciencias Agrarias de la Universidad Nacional de Colombia, Sede Bogotá por la estudiante María del Mar González Trujillo como requisito para obtener el título de Ingeniera Agrónoma para el año 2017. Este proyecto fue financiado por la Convocatoria Nacional de Proyectos para el Fortalecimiento de la Investigación, Creación e Innovación de la Universidad Nacional de Colombia 2016-2018 y dirigido por la profesora Helena Luisa Margarita Brochero y el Ing. agrónomo Andrés Ricardo Peraza Arias.

\section{Contribución de los autores}

María del Mar González-Trujillo: Realización de la colecta de insectos a determinar, toma de datos, realización de mapas y gráficas, interpretación y análisis de resultados mediante la guía de la directora del trabajo de grado y el codirector.

Andrés Ricardo Peraza Arias: Participación activa en la elaboración del manuscrito y su preparación para el sometimiento a la revista en todos sus apartados, al igual que en las posteriores modificaciones propuestas por los pares evaluadores. Determinación de insectos colectados, análisis estadísticos, la estimación de indices ecológicos, abundancia relativa y su asociación con variables fenológicas.

Helena Luisa Margarita Brochero: Dirección de la investigación y coordinación de todas las actividades desarrolladas durante el estudio. Participación en el análisis de la información y en la elaboración del manuscrito. 